\title{
Tyrosine Kinase Inhibitor Sequencing in Patients with Chronic Myeloid Leukemia
}

\author{
Mario Tiribelli $\cdot$ Ahmet Emre Eskazan
}

Received: June 20, 2019 / Published online: August 8, 2019

(C) The Author(s) 2019

\begin{abstract}
The management of chronic myeloid leukemia (CML) has been revolutionized by the discovery of tyrosine kinase inhibitors (TKIs) against $B C R$ $A B L 1$ oncogenic fusion protein. Imatinib, the first $B C R-A B L 1$ TKI, was introduced into clinical practice in the early 2000s. In the following years, the so-called second-generation TKIs (2GTKIs) - dasatinib, nilotinib, and bosutinib were approved, initially for patients resistant to imatinib, and subsequently for front-line treatment. With multiple TKIs available, selection of first-line therapy is challenging. CML risk, patient characteristics and potential toxicities of different TKIs play a fundamental role, in particular when deciding between imatinib and 2GTKIs as frontline treatment. So, when
\end{abstract}

Mario Tiribelli and Ahmet Emre Eskazan contributed equally to the manuscript.

Enhanced Digital Features To view enhanced digital features for this article go to https://doi.org/10.6084/ m9.figshare.9007847.

\section{Tiribelli}

Division of Hematology and BMT, Department of Medical Area, University of Udine, Udine, Italy

\section{A. E. Eskazan $(\bowtie)$}

Division of Hematology, Department of Internal Medicine, Cerrahpasa Faculty of Medicine, Istanbul University-Cerrahpasa, Istanbul, Turkey

e-mail: emreeskazan@hotmail.com; emre.eskazan@istanbul.edu.tr deciding front-line therapy for a patient with CML in the chronic phase (CML-CP), clinicians must consider both the long-term outcomes, such as overall survival and progression-free survival, as well as safety, tolerance and possible treatment discontinuation. This paper offers a practical algorithmic approach for the sequential use of commercially available TKIs in patients with CML-CP along with the data available in the literature.

Keywords: Bosutinib; Chronic myeloid leukemia; Dasatinib; Imatinib; Ponatinib; Tyrosine kinase inhibitor

\section{INTRODUCTION}

The management of chronic myeloid leukemia (CML) has been revolutionized by the discovery of tyrosine kinase inhibitors (TKIs) against BCR$A B L 1$ oncogenic fusion protein. Imatinib, the first $B C R-A B L 1$ TKI, was introduced into clinical practice in the early 2000s. In the following years, the so-called second-generation TKIs (2GTKIs)—dasatinib, nilotinib, and bosutinib were approved, initially for patients resistant to imatinib and subsequently for front-line treatment. These agents induce more rapid and profound responses compared to imatinib but, at present, this has not translated into superior long-term survival benefit, and 2GTKIs can be 
associated with non-hematologic toxicities that may cause concern [1-3].

So, when deciding front-line therapy for a patient with CML in chronic phase (CML-CP), clinicians must consider both the long-term outcomes, such as overall survival (OS) and progression-free survival (PFS), as well as safety, tolerance and possible treatment discontinuation. A recent multicenter observational study performed in Italy on more than 1000 newly diagnosed CML patients found that $48.5 \%$ and $51.4 \%$ of patients were treated frontline imatinib and 2GTKIs, respectively [4].

In this paper, we seek to offer a practical algorithmic approach for the sequential use of commercially available TKIs in patients with CML-CP, using the data available in the literature.

This article is based on previously conducted studies and does not contain any studies with human participants or animals performed by any of the authors.

\section{IMATINIB AS FIRST-LINE THERAPY}

Imatinib now has an almost two-decade long history in the treatment of CML. Updated follow-up of the pivotal International Randomized Study of Interferon Vs STI571 (IRIS) trial has demonstrated that estimated OS at 10 years was $83.3 \%$ with a rate of complete cytogenetic response (CCyR) over 80\% [5]. Similar results have been reported in the German CML-study IV that included more than 1500 patients treated with 5 different imatinib-based frontline regimens [6]. This latter study also found that survival was similar between the standard (i.e. imatinib $400 \mathrm{mg} /$ day) and the experimental arms, and life expectancy of patients with optimal response to therapy was close to that of healthy individuals. More recently, a "real-life" experience from three French reference centers on $418 \mathrm{CML}$ patients receiving front-line imatinib $400 \mathrm{mg}$ also confirmed excellent OS rates (91\% and $82 \%$ at 5 and 10 years) in patients treated outside the restriction of a clinical trial and with minimal loss of follow-up [7].

Taken together, these studies support the choice of imatinib $400 \mathrm{mg}$ as front-line therapy for CML patients, maybe with the exclusion of those with a high-risk Sokal score (Fig. 1). Nonetheless, a significant proportion of CML patients treated with imatinib discontinue therapy sooner or later, with $20-30 \%$ of cases requiring a switch to a different TKI for resistance or intolerance. The excellent rates of OS and progression-free survival (PFS) reported in many studies are, by all evidence, proof of the efficacy of 2GTKI as salvage therapy.

Dasatinib and nilotinib have been used for the treatment of CML patients failing imatinib, either for resistance or intolerance, for more than 10 years. The 7 -year update of the phase 3 , dose-optimization study of dasatinib in 670 CML-CP patients resistant or intolerant to imatinib confirmed that $100 \mathrm{mg}$ daily is the best dose, with PFS and OS of $42 \%$ and $65 \%$ respectively, a major molecular response (MMR) rate of $46 \%$ and an acceptable safety profile, with drug-related pleural effusion rate of $28 \%$ and $<5 \%$ of pulmonary hypertension and arterial ischemic events [8]. With a shorter follow-up, nilotinib has shown similar results both in a phase 2 study (CCyR rate at 48 months of $45 \%$, estimated PFS and OS at 48 months $57 \%$ and $78 \%$, respectively) [9], and in a French reallife experience $(66 \%$ patients achieved MMR and $44 \%$ achieved a deep molecular response (DMR) after a median time of nilotinib therapy around 6 months; survival not reported but only $1 / 146$ patients progressed to advanced phase) [10]. More recently, an Italian group retrospectively analyzed 163 patients treated with 2GTKI (dasatinib, $n=95$ or nilotinib, $n=68$ ) after imatinib failure, reporting rates of CCyR (75\%), MMR (60\%) and DMR (37\%) higher than previous studies, 5-year PFS and OS around $85 \%$, good persistence of treatment (60\% at 4 years) and comparable efficacy and safety of the two TKIs [11].

For CML-CP patients failing imatinib and dasatinib or nilotinib, long-term prognosis could still be fairly good, mainly in terms of survival. Switching to other 2GTKIs has provided rates of CCyR and MMR of $40 \%$ and $16 \%$, respectively, and a median OS of 46 months [12]. In addition to that, a recent study focusing on the use of dasatinib/nilotinib as third-line treatment with a median of 12 months (range 


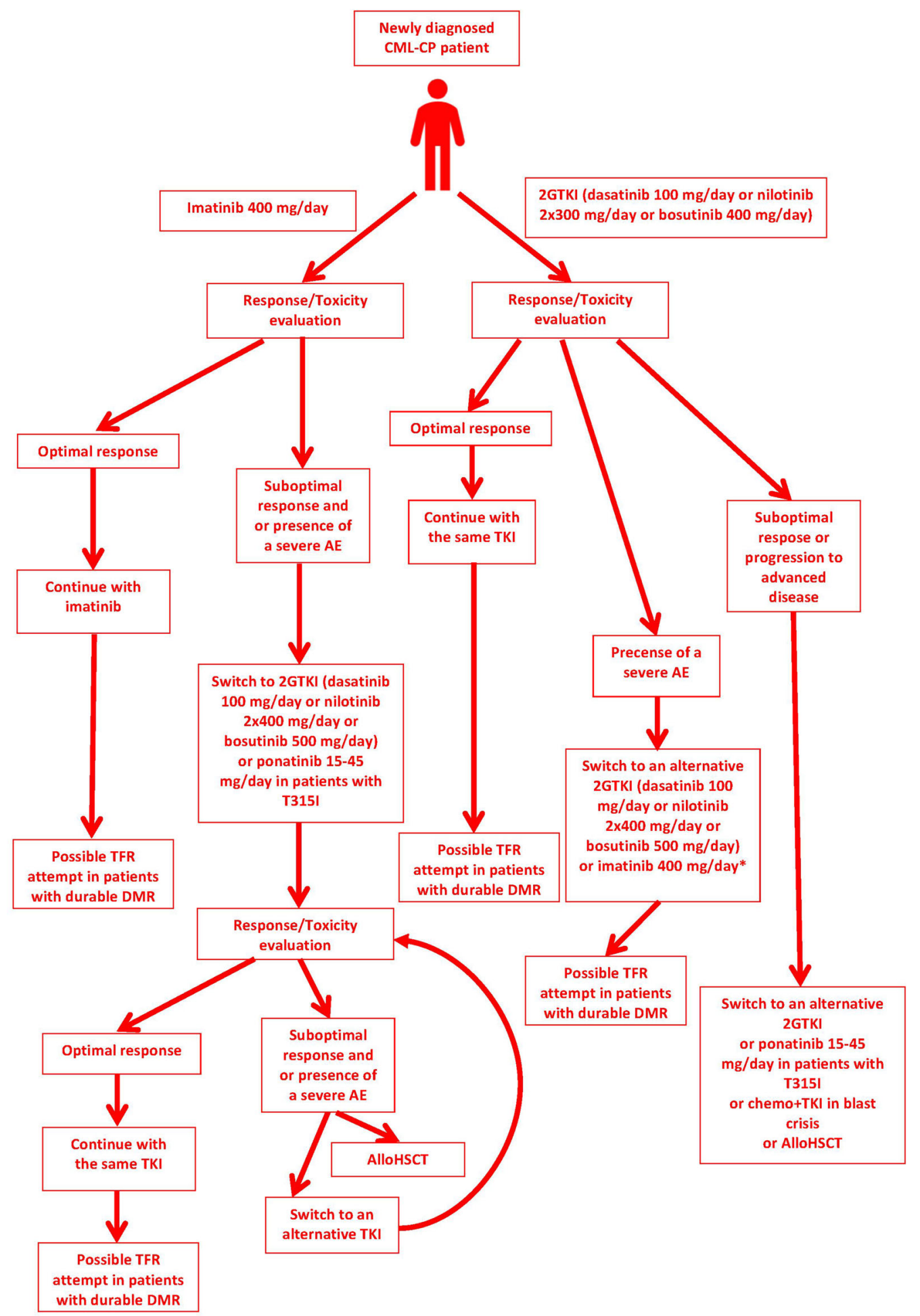

Fig. 1 An algorithmic treatment approach to a newly diagnosed patient with chronic myeloid leukemia in chronic phase (CML-CP). 2GTKI second-generation tyrosine kinase inhibitor, $A E$ adverse event, AlloHSCT allogeneic hematopoietic stem cell transplantation, $D M R$ deep molecular response, TFR treatment-free remission. *See text for details 
1-42 months) after failure of two previous TKIs showed that 16 out of 21 patients (76\%) in this setting were able to gain and/or maintain an optimal molecular response [13]. A significant breakthrough for patients with resistance or intolerance to multiple TKIs has come with the availability of the so-called "third generation" TKI-ponatinib. Updated 5-year data from the phase 2 Ponatinib $\mathrm{Ph}^{+}$ALL and CML Evaluation (PACE) trail on 267 CP-CML patients treated with ponatinib after failure of multiple TKIs (most patients have been treated with three or four prior drugs) reported cumulative rates of CCyR and MMR of 54\% and 40\%, respectively, with $24 \%$ of deep MR, being 5-year PFS and OS $49 \%$ and $77 \%$ [14]. Ponatinib is thus emerging as the most effective treatment in patients failing imatinib and at least one 2GTKI, but the starting dose of $45 \mathrm{mg}$ is associated with a significant risk of cardiovascular adverse event. Thus, various trials and retrospective experiences are testing the efficacy and safety of lower doses of ponatinib, either as starting dose or as a reduction strategy following attainment of response [15].

\section{GTKIs AS FIRST-LINE THERAPY}

As 2GTKIs are more potent than imatinib, many physicians tend to use these agents in the upfront setting among patients with CML-CP, since these newer TKIs have the ability to induce faster [early molecular response (EMR)] and deeper (DMR) responses [16]. Three 2GTKIs (bosutinib, dasatinib, and nilotinib) have demonstrated significant clinical benefit compared with imatinib in randomized clinical trials [17-19], and although not always available worldwide, these agents are approved in the management of newly diagnosed patients with CML in some countries.

The choice of selecting any 2GTKI over imatinib as an upfront treatment option depends on various factors, mainly the disease risk factors, comorbidities, and the willingness of the patient for a treatment-free remission (TFR) attempt, especially if the patient is young, since the probability of achieving a sustained DMR is higher with 2GTKIs than with imatinib.
When a patient with CML-CP has started any 2GTKI as upfront treatment, there are three possible scenarios: (1) the patient may do perfectly well under the particular 2GTKI both in terms of efficacy and toxicity, so he/she will continue receiving the same TKI; (2) the patient needs to quit the 2GTKI due to toxicity, regardless of response situation under the same TKI (optimal or suboptimal); (3) the patient might discontinue the currently administered 2GTKI due to suboptimal response (both primary and secondary resistance) or progression (e.g. from CP to advanced phases) (Fig. 1).

In the first scenario, the optimal responder could continue the current 2GTKI, unless there would be a TFR attempt, as in case of sustained DMR [20]. There are discontinuation studies with dasatinib and nilotinib but not yet with bosutinib [21]. Moreover, nilotinib was recently licensed in the context of TFR in the United States, when used either in the upfront or salvage settings for at least 3 years with a durable (for at least 24 months) DMR (defined as $\mathrm{MR}^{4.5}$ ) status [16]. Although percentages may vary from study to study, approximately $40-50 \%$ of the cases who attempted a TFR will lose their molecular responses, and reinitiation of the former TKI could be necessary. After restarting the same TKI, nearly all patients regain at least optimal molecular response $\left(\mathrm{MR}^{3}\right)$ or even DMR [21].

The second scenario, which is the permanent discontinuation of the 2GTKI due to adverse events (AEs), may result in two different outcomes. First, the patient might have an optimal response prior to quitting the TKI, then there would be no need of an immediate TKI therapy or, if a subsequent TKI therapy is planned, the patient may receive either imatinib or an alternative 2 GTKI. Second, if the patient stopped the first-line 2GTKI treatment due to toxicity prior to achieving an optimal response, the remaining 2GTKIs can be the treatment of choice and, in addition to that, imatinib can still be an option. However, in this particular case, where a 2GTKI was preferred over imatinib at diagnosis, most probably the patient would want to attempt TFR in the future, and/or the patient might have a high-risk disease, so continuing with another 2GTKI would be more logical. 
In the third scenario, where the patient has stopped the 2GTKI therapy due to resistance, the remaining two 2GTKIs can be chosen according to the ABL kinase domain mutational status of the patient and/or the comorbidities should also be taken into consideration. In the case of a T315I mutation, ponatinib should be the treatment of choice. If a patient progresses to blast crisis, the patient should be checked for mutations, and appropriate induction chemotherapy together with a TKI should be initiated. If this therapy can induce a second $\mathrm{CP}$, allogeneic hematopoietic stem cell transplantation (AlloHSCT) should also be kept in mind for the available patients, and patients who have failed two lines of TKI therapy should also be evaluated for AlloHSCT as well [22].

The availability of five different TKIs, of which four are approved for the newly diagnosed patient, can make the selection of an appropriate frontline therapy challenging. Individual disease and patient characteristics, such as risk of CML progression, age, concomitant disease or medications and patient's expectations may guide physicians in the choice. However, besides the TKI chosen for first-line (either imatinib or a 2GTKI), a careful and timely monitoring, with prompt switch to alternative therapy in case of non-optimal response or intolerance, may grant CML patients a nearly normal life expectancy and excellent quality of life.

\section{ACKNOWLEDGEMENTS}

Funding. No funding or sponsorship was received for this study or publication of this article.

Authorship. All named authors meet the International Committee of Medical Journal Editors (ICMJE) criteria for authorship for this article, take responsibility for the integrity of the work as a whole, and have given their approval for this version to be published.

Disclosures. Mario Tiribelli has received speaker bureau and advisory board honoraria from Novartis, Bristol-Myers Squibb, Pfizer, and Incyte, outside the present study. Ahmet Emre Eskazan has received advisory board honorarium from Novartis, and he also received speaker bureau honoraria from Novartis, Bristol-Myers Squibb, and Pfizer, outside the present study.

Compliance with Ethics Guidelines. This article is based on previously conducted studies and does not contain any studies with human participants or animals performed by any of the authors.

Open Access. This article is distributed under the terms of the Creative Commons Attribution-NonCommercial 4.0 International License (http://creativecommons.org/licenses/ by-nc/4.0/), which permits any noncommercial use, distribution, and reproduction in any medium, provided you give appropriate credit to the original author(s) and the source, provide a link to the Creative Commons license, and indicate if changes were made.

\section{REFERENCES}

1. Cortes JE, Saglio G, Kantarjian HM, et al. Final 5-year study results of DASISION: the dasatinib versus imatinib study in treatment-naïve chronic myeloid leukemia patients trial. J Clin Oncol. 2016;34:2333-40.

2. Hochhaus A, Saglio G, Hughes TP, et al. Long-term benefits and risks of frontline nilotinib vs imatinib for chronic myeloid leukemia in chronic phase: 5 -year update of the randomized ENESTnd trial. Leukemia. 2016;30:1044-54.

3. Eskazan AE, Ozmen D. Tyrosine kinase inhibitor (TKI) therapy for newly-diagnosed patients with chronic myeloid leukemia: focusing on TKI discontinuation due to adverse events-is better always good? Expert Rev Hematol. 2017;10(7):583-6.

4. Specchia G, Pregno P, Nicolosi M, et al. Chronic Myeloid Leukemia Italian Multicenter Observational Study (CML-IT-MOS): clinical characteristics of chronic myeloid leukemia (CML) patients treated in real-life between 2012 and 2016 in 66 Italian Hematology Centers of the Gimema Study Group. Blood. 2018;132:45 (abstract). 
5. Hochhaus A, Larson RA, Guilhot F, et al. Long-term outcomes of imatinib treatment for chronic myeloid leukemia. N Engl J Med. 2017;376:917-26.

6. Hehlmann R, Lauseker M, Saussele S, et al. Assessment of Imatinib as first-line treatment of chronic myeloid leukemia: 10-year survival results of the randomized CML study IV and impact of non-CML determinants. Leukemia. 2017;31:2398-406.

7. Nicolini FE, Alcazer V, Cony-Makhoul P, et al. Long-term follow-up of de novo chronic phase chronic myelogenous leukemia patients on frontline imatinib. Exp Hematol. 2018;64:97-105.

8. Shah NP, Rousselot P, Schiffer C, et al. Dasatinib in imatinib-resistant or -intolerant chronic-phase, chronic myeloid leukemia patients: 7-year followup of study CA180-034. Am J Hematol. 2016;91:869-74.

9. Giles FJ, le Coutre PD, Pinilla-Ibarz J, et al. Nilotinib in imatinib-resistant or imatinib-intolerant patients with chronic myeloid leukemia in chronic phase: 48-month follow-up results of a phase II study. Leukemia. 2013;27:107-12.

10. Cony-Makhoul P, Gardembas M, Coiteux V, et al. Nilotinib after imatinib first-line: a real-life longitudinal cohort of patients with chronic myeloid leukaemia in chronic phase. $\mathrm{Br} \mathrm{J}$ Haematol. 2018;180:356-64.

11. Tiribelli M, Bonifacio M, Binotto G, et al. Excellent outcomes of 2G-TKI therapy after imatinib failure in chronic phase CML patients. Oncotarget. 2018;9:14219-27.

12. Russo Rossi A, Breccia M, Abruzzese E, et al. Outcome of 82 chronic myeloid leukemia patients treated with nilotinib or dasatinib after failure of two prior tyrosine kinase inhibitors. Haematologica. 2013;98:399-403.

13. Ongoren S, Eskazan AE, Suzan V, et al. Third-line treatment with second-generation tyrosine kinase inhibitors (dasatinib or nilotinib) in patients with chronic myeloid leukemia after two prior TKIs: reallife data on a single center experience along with the review of the literature. Hematology. 2018;23(4):212-20.
14. Cortes JE, Kim DW, Pinilla-Ibarz J, et al. Ponatinib efficacy and safety in Philadelphia chromosomepositive leukemia: final 5-year results of the phase 2 PACE trial. Blood. 2018;132:393-404.

15. Molica M, Scalzulli E, Colafigli G, et al. Insights into the optimal use of ponatinib in patients with chronic phase chronic myeloid leukaemia. Ther Adv Hematol. 2019;10:1-14.

16. Eşkazan AE. Evolving treatment strategies in CMLmoving from early and deep molecular responses to TKI discontinuation and treatment-free remission: is there a need for longer-term trial outcomes? $\mathrm{Br} \mathrm{J}$ Clin Pharmacol. 2018;84(8):1635-8.

17. Cortes JE, Gambacorti-Passerini C, Deininger MW, et al. Bosutinib versus imatinib for newly diagnosed chronic myeloid leukemia: results from the randomized BFORE Trial. J Clin Oncol. 2018;36(3):231-7.

18. Cortes JE, Saglio G, Kantarjian HM, et al. Final 5-year study results of DASISION: the dasatinib versus imatinib study in treatment-naïve chronic myeloid leukemia patients trial. J Clin Oncol. 2016;34(20):2333-40.

19. Hochhaus A, Saglio G, Hughes TP, et al. Long-term benefits and risks of frontline nilotinib vs imatinib for chronic myeloid leukemia in chronic phase: 5 -year update of the randomized ENESTnd trial. Leukemia. 2016;30(5):1044-54.

20. Saussele S, Richter J, Guilhot J, EURO-SKI investigators, et al. Discontinuation of tyrosine kinase inhibitor therapy in chronic myeloid leukaemia (EURO-SKI): a prespecified interim analysis of a prospective, multicentre, non-randomised, trial. Lancet Oncol. 2018;19(6):747-57.

21. Cortes J, Rea D, Lipton JH. Treatment-free remission with first- and second-generation tyrosine kinase inhibitors. Am J Hematol. 2019;94(3):346-57.

22. Eskazan AE, Tiribelli M. Allogeneic haematopoietic stem cell transplantation for chronic myeloid leukaemia in the era of tyrosine kinase inhibitors. EMJ Hematol. 2018;6(1):63-70. 